\title{
Abbreviations
}

$\begin{array}{ll}\text { AAVE } & \text { African American Vernacular English } \\ \text { AbE/C/P } & \text { (Australian) Aboriginal English / Creole / Pidgin } \\ \text { AfBahE } & \text { Afro-Bahamian English } \\ \text { AfkE } & \text { Afrikaans English } \\ \text { AmE } & \text { American English } \\ \text { AnBahE } & \text { Anglo-Bahamian English } \\ \text { AppE } & \text { Appalachian English } \\ \text { AusE/VE/C } & \text { Australian English/Vernacular English/Creoles } \\ \text { BahE } & \text { Bahamian English } \\ \text { Baj } & \text { Bajan (Barbadian Creole) } \\ \text { BelC } & \text { Belizean Creole } \\ \text { BIE } & \text { Bay Islands English (Honduras) } \\ \text { BrC } & \text { British Creole } \\ \text { BrE } & \text { British English (= EngE }+ \text { ScE }+ \text { WelE) } \\ \text { ButlE } & \text { Butler English (India) } \\ \text { CajE } & \text { Cajun English } \\ \text { CAmC } & \text { Central American Creoles (Belize, Miskito, Limón, etc.) } \\ \text { CamP/E } & \text { Cameroon Pidgin/English } \\ \text { CanE } & \text { Canadian English } \\ \text { CarE } & \text { Caribbean English } \\ \text { Car(E)C } & \text { Carribean (English-lexicon) Creoles } \\ \text { CFE } & \text { Cape Flats English } \\ \text { ChcE } & \text { Chicano English } \\ \text { ChnP } & \text { Chinese Pidgin English } \\ \text { CollAmE } & \text { Colloquial American English } \\ \text { CollSgE } & \text { Colloquial Singapore English } \\ \text { EAfE } & \text { East African English } \\ \text { EMarC } & \text { Eastern Maroon Creole } \\ \text { EngE } & \text { English English } \\ \text { EModE } & \text { Early Modern English } \\ \text { ME } & \text { Middle English } \\ \text { OE } & \text { Old English } \\ \text { ESM } & \text { English in Singapore and Malaysia } \\ \text { FijE } & \text { Fiji English } \\ \text { GhE/P } & \text { Ghanaian English/Pidgin } \\ \text { GuyC } & \text { Guyanese Creole } \\ \text { HawC } & \text { Hawaii Creole } \\ \text { HKE } & \text { Hong Kong English } \\ \text { IndE } & \text { Indian English, Anglo-Indian } \\ & \end{array}$




$\begin{array}{ll}\text { InlNE } & \text { Inland Northern (American) English } \\ \text { IrE } & \text { Irish English } \\ \text { JamC/E } & \text { Jamaican Creole / English } \\ \text { KenE } & \text { Kenyan English } \\ \text { KPE } & \text { Kru Pidgin English } \\ \text { LibC/E } & \text { Liberian Creole/English } \\ \text { LibSE } & \text { Liberian Settler English } \\ \text { LibVE } & \text { Liberian Vernacular English } \\ \text { LimC } & \text { Limonese Creole (Costa Rica) } \\ \text { LonVE } & \text { London Vernacular English } \\ \text { LnkE } & \text { Lankan English } \\ \text { MalE } & \text { Malaysian English } \\ \text { NEngE } & \text { New England English } \\ \text { NfldE } & \text { Newfoundland English } \\ \text { NigP/E } & \text { Nigerian Pidgin / English } \\ \text { NZE } & \text { New Zealand English } \\ \text { NYCE } & \text { New York City English } \\ \text { OzE } & \text { Ozarks English } \\ \text { PakE } & \text { Pakistani English } \\ \text { PanC } & \text { Panamanian Creole } \\ \text { PhilE } & \text { Philadelphia English } \\ \text { PhlE } & \text { Philippines English } \\ \text { RP } & \text { Received Pronunciation } \\ \text { SAfE } & \text { South African English } \\ \text { BlSAfE } & \text { Black South African English } \\ \text { CoSAfE } & \text { Coloured South African English } \\ \text { InSAfE } & \text { Indian South African English } \\ \text { WhSAfE } & \text { White South African English } \\ \text { SAmE } & \text { Southern American English } \\ \text { SAsE } & \text { South Asian English } \\ \text { SEAmE } & \text { South Eastern American English enclave dialects } \\ \text { ScE } & \text { Scottish English, Scots } \\ \text { ScStE } & \text { Scottish Standard English } \\ \text { SgE } & \text { Singapore English } \\ \text { SLVE } & \text { St. Lucian Vernacular English } \\ \text { SolP } & \text { Solomon Islands Pidgin } \\ \text { StAmE } & \text { Standard American English } \\ \text { StAusCE } & \text { Standard Australian Colloquial English } \\ \text { StAusFE } & \text { Standard Australian Formal English } \\ \text { StBrE } & \text { Standard British English } \\ \text { StE } & \text { Standard English } \\ \text { StGhE } & \text { Standard Ghanaian English } \\ & \text { Stan } \\ \end{array}$


StHE St. Helena English

StIndE Standard Indian English

StJamE Standard Jamaican English

SurC Suriname Creoles

TanE Tanzanian English

TobC Tobagonian Creole

Trad-RP Traditional Received Pronunciation

TrnC Trinidadian Creole

T\& TC Trinidadian \& mesolectal Tobagonian Creoles

TP Tok Pisin, New Guinea Pidgin, Neomelanesian

WAfE/P West African English/Pidgin

WelE Welsh English

WMwE Western and Midwestern American English

ZamE Zambian English

\section{More abbreviations}

$\begin{array}{ll}\text { ESL } & \text { English as Second Language } \\ \text { EFL } & \text { English as Foreign Language } \\ \text { EIL } & \text { English as International Language } \\ \text { ENL } & \text { English as Native Language } \\ \text { L1 } & \text { First Language } \\ \text { L2 } & \text { Second Language } \\ \text { P/C } & \text { Pidgins and Creoles }\end{array}$


